\title{
Resistência à compressão de argamassas em função da adição de fibra de coco
}

\author{
Everton J. da Silva ${ }^{1}$, Paola D. da Silva ${ }^{2}$, Maria L. Marques ${ }^{3}$, \\ Celso C. M. Fornari Junior ${ }^{4}$, Fermin C. Garcia ${ }^{5}$ \& Francisco H. M. Luzardo 6 \\ ${ }^{1}$ IFBA Eunápolis, Eunápolis, BA. E-mail: js_everton@yahoo.com.br. Bolsista FAPESB (Autor correspondente) \\ ${ }^{2}$ IFBA Eunápolis, Eunápolis, BA. E-mail: paola.dias@hotmail.com.br. Bolsista PIBIC-EM \\ ${ }^{3}$ IFBA Eunápolis, Eunápolis, BA. E-mail: lidiane_marques@yahoo.com.br. Bolsista FAPESB \\ ${ }^{4}$ DCET/UESC, Ilhéus, BA. E-mail: celso@uesc.br \\ ${ }^{5}$ DCET/UESC, Ilhéus, BA. E-mail: fermingv@gmail.com \\ ${ }^{6}$ DCET/UESC, Ilhéus, BA. E-mail: fmartinezluzardo@gmail.com
}

\section{Palavras-chave:}

fibras naturais

matriz cimentícia

compósito

\begin{abstract}
R E S U M O
Neste trabalho se propôs analisar a influência que a variação do comprimento da fibra de coco exerce na resistência à compressão da argamassa, em busca de se determinar, experimentalmente, o comprimento mais próximo ao tamanho crítico. Foram confeccionadas argamassas com adição $(0,3 \%$ em relação ao volume total da mistura) de 6 comprimentos diferentes de fibras de coco: 12,5; 25,0; 37,5; 50,0; 62,5 e 75,0 mm, além de uma argamassa de referência sem adição de fibra de coco. Para a avaliação do comportamento de cada um deles na argamassa foram efetuados ensaios de consistência no estado fresco e resistência à compressão axial no estado endurecido, realizado nas idades 7, 28 e 56 dias, ao longo do primeiro semestre de 2013. Foi observado que todos os comprimentos de fibra testados diminuem a resistência à compressão em relação à argamassa de referência, nas três idades. No entanto, dentre esses comprimentos testados, pode-se afirmar que a argamassa com adição de fibras de comprimento 25,0 $\mathrm{mm}$ obteve melhor desempenho nas três idades testadas.
\end{abstract}

Key words: natural fibers cement matrix composite

\section{Compressive strength of cement mortar prepared with the addition of coconut fiber}

\begin{abstract}
A B S T R A C T
This paper aims to analyse the influence that the length of coconut fiber exerts on compressive strength of mortar seeking to determine their critical length. Mortars were prepared with addition ( $0.3 \%$ volume of the mixture) of 6 different lengths of coconut fibers: $12.5,25.0,37.5,50.0,62.5$ and $75.0 \mathrm{~mm}$, and a reference mortar. To study the behavior of each one in mortar, consistency tests were performed on fresh and hard state in axial compression strength test at three ages (7, 28 and 56 days) during the first half of 2013. It was observed that all lengths tested decrease resistance compared to the reference mortar in three ages, however, the mortar with addition of $25.0 \mathrm{~mm}$ length fibers had a better performance in the study.
\end{abstract}

\section{INTRODUÇÃO}

As fibras naturais existem em abundância e têm seu uso motivado por serem renováveis, biodegradáveis e pela sua disponibilidade a baixo custo. Muitas vezes como resíduos e sem uma destinação específica, as fibras acabam se transformando em problemas de ordem ambiental. Cabe, portanto, aos pesquisadores, a responsabilidade de encontrar soluções viáveis do ponto de vista econômico, ecológicas do ponto de vista ambiental e eficazes do ponto de vista tecnológico.

No Brasil se destaca, dentre os processos agroindustriais necessários para o desenvolvimento da sociedade, principalmente pela busca de alimentos mais saudáveis, a cultura do coco cujo produto principal é a água de coco para consumo interno e exportação.
De acordo com o IBGE (2013), através do LSPA (Levantamento Sistemático da Produção Agrícola), a produção de coco no Brasil alcançou 1.932.504 de toneladas na safra 2013. Considerando que aproximadamente $10 \%$ da massa do fruto são constituídos por fibras, o volume gerado em 2013 foi em torno de $966.252 \mathrm{~m}^{3}$ de fibra de coco.

Esta grande e crescente quantidade de coco propicia a oferta e a utilização de suas fibras para diversas finalidades, dentre elas a incorporação (através de adição) em matrizes de cimento Portland vem sendo estudada visto que diversas pesquisas recentes, como as realizadas por Jhon et al. (2005), Gunasekaran et al. (2011), Rodríguez et al. (2011), Ramires et al. (2012), Silva et al. (2012), Ramli et al. (2013) e Pereira et al. (2013), apontam que sua aplicação pode melhorar as propriedades físicas e mecânicas de compósitos cimentícios. 
Além desses trabalhos, outros autores, como Savastano Júnior et al. (1999), Toledo Filho et al. (2000; 2003), Brígida et al. (2010), Abdullah et al. (2011) e Claramunt et al. (2011), discutem como a degradação da fibra de coco ocorre e quais os possíveis dispositivos para tentar mitigá-la. Esses estudos indicam que sistemas de tratamento da fibra com ciclos de lavagem e utilização de revestimentos têm-se mostrado promissores para se tornar uma metodologia adequada na prevenção da fibra, frente ao ataque alcalino cimentício.

Um aspecto importante na incorporação da fibra de coco é a influência que o comprimento da fibra de coco exerce no comportamento do compósito. Silva Filho \& Garcez (2007) afirmam que os mecanismos de transferência de tensões num compósito são influenciados pelo comprimento "crítico" das fibras, que é o menor comprimento necessário para o seu desenvolvimento de tensões máximas.

Quando o comprimento da fibra é menor do que o crítico, a ancoragem não é suficiente para gerar tensões de escoamento ou de ruptura nas fibras e, consequentemente, serão sacadas da matriz, caso em que as fibras não estarão atuando de forma eficiente no sentido de fornecer uma melhora nas propriedades do compósito, como resistência e tenacidade.

Se, entretanto, a fibra apresentar comprimento muito superior ao comprimento crítico, a resistência do compósito começará a diminuir devido às falhas provenientes do emaranhamento das fibras e aglomerações originárias durante o processamento (Hassan et al., 2010).

Ainda de acordo com Silva Filho \& Garcez (2007), para o caso de fibras menos resistentes, como as de coco, a ruptura da fibra pode envolver menor consumo de energia que o seu arrancamento e, neste caso, ocorrerá redução da energia total envolvida no processo de ruptura do compósito, tornando o material mais frágil e facilitando seu rompimento. A máxima tenacidade deste tipo de compósito é obtida quando o comprimento médio das fibras empregadas é igual ao comprimento crítico.

Ali et al. (2012) estudaram as propriedades dinâmicas e estáticas de concretos com teores de adição de fibra que variaram de 1 a $5 \%$ (em relação à massa do cimento) cujos comprimentos foram: 25,0; 50,0 e 75,0 mm. De acordo com os resultados obtidos, o comprimento de 50,0 $\mathrm{mm}$ obteve os melhores resultados em sua pesquisa.

Ali et al. (2013) porém, testaram, em busca de avaliar a força de ligação entre a fibra de coco e o concreto, o efeito do comprimento das fibras de coco, seus diâmetros, condições de pretratamento e proporções de mistura para o concreto. Referente ao comprimento, os autores chegaram à conclusão de que a força de adesão aumenta quando o comprimento da fibra é de 30,0 mm.

Verificam-se, a partir dos trabalhos publicados, questões em aberto relacionadas à incorporação da fibra de coco na matriz cimentícia, como o comprimento das fibras que otimiza seu desempenho, ante o que se objetiva, no presente trabalho, estudar a variação que o comprimento da fibra de coco in natura provoca na consistência e resistência à compressão da argamassa de cimento Portland.

\section{Material e Métodos}

Com base nos objetivos do trabalho e buscando analisar a real interatividade entre a fibra e a matriz, o trabalho experimental se iniciou com a investigação das características dos materiais empregados.

Todos os ensaios deste trabalho foram realizados no Laboratório Interdisciplinar de Mecânica dos Solos e Materiais de Construção - LISMAC, do IFBA/BA, Campus Eunápolis, durante o primeiro semestre de 2013. A fibra de coco foi fornecida pelo Laboratório de Polímeros e Sistemas - LAPOS, UESC/BA.

O cimento Portland utilizado nesta pesquisa foi o CP V ARI RS em virtude da sua maior velocidade de hidratação e menor teor de adições minerais que poderiam influenciar no desempenho da fibra de coco. A água potável usada para confecção dos compósitos foi obtida da rede pública de distribuição da cidade de Eunápolis, Bahia. O agregado miúdo utilizado, procedente da cidade de Eunápolis, foi caracterizado através dos ensaios de granulometria, massa específica real e massa específica aparente, conforme suas respectivas normas.

Para a mistura da argamassa foi utilizada uma argamassaderia eletro-mecânica, marca Contenco, modelo I 3010. A sequência de colocação dos materiais se deu conforme a NBR-7215 (ABNT, 1996). Após a mistura foi efetuado o ensaio de consistência no estado fresco, através do seu abatimento na mesa de consistência sendo que, quanto maior o diâmetro da argamassa obtido na mesa de consistência mais fluido é seu comportamento no estado fresco.

Depois da moldagem os corpos-de-prova (cp's) foram cobertos com um filme plástico. Com $24 \mathrm{~h}$ de cura ao ar livre foram realizadas a desforma e a colocação dos cp's na câmara úmida até o momento do ensaio de resistência à compressão axial no estado endurecido, realizado na prensa eletrohidráulica, marca Contenco, modelo I $3025 \mathrm{~B}$, de acordo com a NBR-7215 (ABNT, 1996).

Com base nas recomendações de Toledo Filho (1997), que sugere utilizar um teor de adição de fibra de coco entre 0,1 a $0,5 \%$ em relação ao volume do compósito, foi utilizado o teor de adição de 0,3\%; na dosagem da argamassa utilizou-se o traço 1:2,3 (cimento:areia) e com relação água/aglomerante 0,55 . Este traço e a relação água/aglomerante foram definidos a partir do estudo de Li et al. (2006), que proporcionou consistência adequada e trabalhabilidade à mistura. Estudaram-se 7 tipos de misturas: sem adição de fibras (referência) e com adição de fibras de coco (FC) utilizando os seguintes comprimentos: 12,5; 25,$0 ; 37,5 ; 50,0 ; 62,5$ e $75,0 \mathrm{~mm}$; as quantidades utilizadas para cada traço estão dispostas na Tabela 1 .

Tabela 1. Dosagem da argamassa

\begin{tabular}{cccccc}
\hline \multirow{2}{*}{$\begin{array}{c}\text { Mistura da } \\
\text { argamassa }\end{array}$} & Traço & \multicolumn{4}{c}{ Consumo de materiais (g) } \\
\cline { 3 - 6 } Referência & $1: 2,3: 0,55$ & 400,00 & 920,00 & 220 & - \\
FC 12,5 mm & $1: 2,3: 0,55$ & 400,00 & 920,00 & 220 & 3,24 \\
FC 25,0 mm & $1: 2,3: 0,55$ & 400,00 & 920,00 & 220 & 3,24 \\
FC 37,5 mm & $1: 2,3: 0,55$ & 400,00 & 920,00 & 220 & 3,24 \\
FC 50,0 mm & $1: 2,3: 0,55$ & 400,00 & 920,00 & 220 & 3,24 \\
FC 62,5 mm & $1: 2,3: 0,55$ & 400,00 & 920,00 & 220 & 3,24 \\
FC 75,0 mm & $1: 2,3: 0,55$ & 400,00 & 920,00 & 220 & 3,24 \\
\hline
\end{tabular}


Conforme a norma NBR-7215 (ABNT, 1996) foi utilizado um desvio relativo máximo de $6 \%$ e o desvio padrão de cada mistura foi calculado. Com base nesta norma, definiram-se as idades de 7, 28 e 56 dias para avaliação da resistência à compressão. Para se conhecer o comportamento das diferenças entre as variáveis utilizou-se o Multiple Range Test de Duncan a um nível de confiabilidade de 0,95 e se fez, na definição dos números de corpos de prova, a opção de 4 cp's para cada idade de cada ensaio, ou seja, 4 repetições para cada tratamento.

\section{Resultados e Discussão}

Em relação aos resultados de caracterização do agregado miúdo utilizado, a análise granulométrica determinou o valor de $1,18 \mathrm{~mm}$ para o diâmetro máximo e 2,49 para o módulo de finura; através dos ensaios de massa específica real e aparente, foram encontrados os valores de 2650 e $1670 \mathrm{~kg} \mathrm{~m}^{-3}$, respectivamente.

Pode-se observar, na Figura 1, que as argamassas com comprimentos de fibras maiores $(50,0 ; 62,5$; e $75,0 \mathrm{~mm})$ apresentaram diminuição da consistência em relação às argamassas com fibras menores e à argamassa de referência. A argamassa FC 75,0 reduziu a consistência 7,4\% em relação à argamassa de referência, o que pode ser atribuído à maior vinculação, absorção de água e ao maior enrolamento que este comprimento de fibra promove, tornando a argamassa com este comprimento de fibra, menos fluida e menos resistente.

Savastano Júnior et al. (1999a) ressaltam que o aumento da quantidade percentual de fibras vegetais está intrinsicamente ligado a uma redução da trabalhabilidade da mistura devido à absorção de água pelas mesmas e ao acréscimo da área superficial de molhagem.

Por outro lado, as argamassas com as fibras de menor comprimento (12,5; 25,0 e 37,5 mm) não prejudicaram a fluidez da argamassa; a argamassa FC 12,5 reduziu a consistência somente $1,75 \%$ em relação à argamassa de referência.

No que diz respeito à resistência à compressão, os resultados da análise de variância mostraram a existência de diferenças significativas entre todos os comprimentos estudados, com uma confiabilidade muito alta, superior a 0,95.

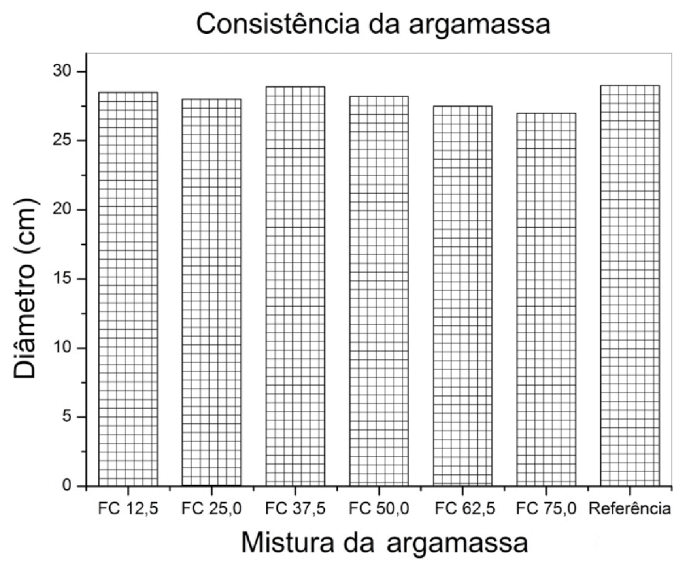

Figura 1. Consistência das argamassas em dependência do comprimento da fibra adicionada
A Figura 2 reúne os valores de resistência à compressão em dependência do comprimento da fibra de coco adicionada; para a idade de 7 dias (Figura 2A), observa-se uma queda da resistência em todas argamassas com adição de fibras de coco em relação à argamassa de referência, sem fibra; as argamassas FC 75,0 e FC 25,0 apresentaram resistência de 26,62 e 28,89 $\mathrm{MPa}$, respectivamente; enquanto isto, a argamassa de referência aparece com 37,23 MPa, valor 39,86\% maior que a argamassa FC 75,0. Verifica-se, na Figura 2B (28 dias) que as argamassas com fibras de menor comprimento $(12,5 ; 25,0$; e $37,5 \mathrm{~mm})$ apresentam desempenho superior às argamassas com fibras de maior comprimento; a argamassa FC 25,0 apresentou resistência 30,01\% maior que a argamassa FC 75,0.

Entende-se, pela análise da Figura 2C (56 dias) que as argamassas adicionadas com fibras de coco de comprimento

A.

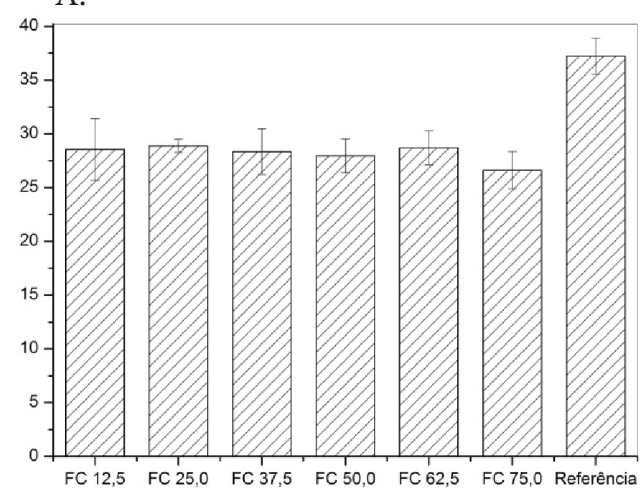

B.

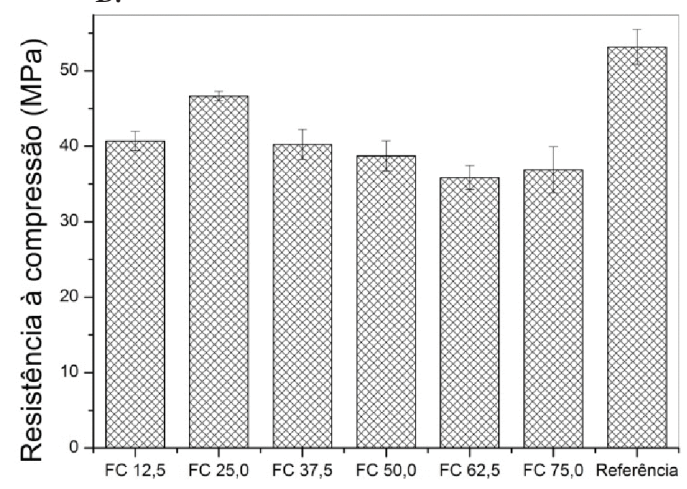

C.

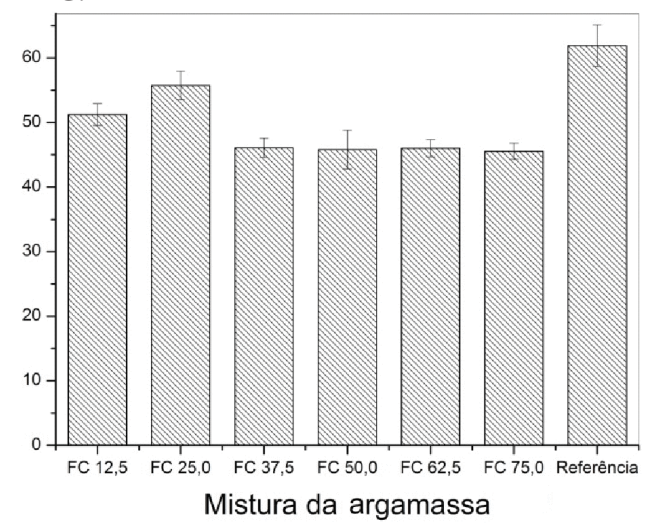

Figura 2. Resistência à compressão aos 7 dias (A), 28 dias (B) e 56 dias (C) em dependência do comprimento da fibra adicionada 
12,5 e 25,0 mm continuaram com melhor desempenho que as argamassas com fibras de comprimentos maiores; a argamassa FC 25,0 foi $22,42 \%$ mais resistente que a argamassa FC 75,0. Constatou-se, ainda em relação à FC 25,0, que a diferença de resistência para argamassa de referência foi de $11,89 \%$ na idade de 56 dias; nesta idade se observa que houve um desempenho muito semelhante entre as argamassas com comprimentos de 37,5; 50,0; 62,5 e 75,0 mm demonstrando que esses comprimentos estão acima do comprimento crítico da fibra de coco.

É possível perceber, pela análise dos resultados de resistência à compressão, que nas condições experimentais testadas, ele deve ser próximo dos 25,0 $\mathrm{mm}$, uma vez que este apresenta desempenho melhor para as três idades avaliadas. Alguns trabalhos recentes (Asasutjarit et al., 2007; Ali et al., 2012; 2013), desenvolvidos em condições distintas, corroboram com esta indicação.

Asasutjarit et al. (2007) pesquisaram a influência que o comprimento da fibra de coco exerce em placas de cimento leve à base de fibra de coco e realizaram os seguintes testes: módulo de ruptura, absorção de água, condutibilidade térmica e resistência à tração. Entre as faixas de comprimento avaliadas ( 1 a $4 \mathrm{~mm}$; 1 a $6 \mathrm{~cm}$ e 1 a $13 \mathrm{~cm}$ ), a considerada ótima foi a intermediária, 1 a $6 \mathrm{~cm}$, indicando que é dentro desta faixa que se encontra o melhor comprimento de ancoragem na matriz cimentícia.

Ali et al. (2012) avaliaram a relação água/aglomerante, consistência, módulo de ruptura, resistência à compressão, resistência à flexão, além de propriedades dinâmicas do concreto reforçado com fibra de coco com 28 dias de idade. Os autores investigaram os teores de 1,2, 3 e 5\% de fibra em relação à massa de cimento cujos comprimentos testados foram 25,50 e $75 \mathrm{~mm}$; ao final do estudo eles concluíram que o compósito com as melhores propriedades foi obtido quando utilizada a fibra de coco com comprimento de aproximadamente 50 $\mathrm{mm}$ e com $5 \%$ de fibra em relação à massa de cimento porém no ensaio de resistência à compressão com $1 \%$ de fibra de coco a diferença de resistência dos concretos com fibras de comprimentos 25 e $50 \mathrm{~mm}$ é de aproximadamente $5 \%$.

Em outro trabalho, Ali et al. (2013) fizeram um estudo aprofundado utilizando a resistência à tração do concreto reforçado com fibra de coco e também da energia de arrancamento necessária para retirar a fibra de coco do concreto com 28 dias de idade; neste exame os autores investigaram o traço do concreto, o comprimento da fibra de coco $(10,20,30$ e $40 \mathrm{~mm})$, o diâmetro da fibra de coco e condições de pré-tratamento das fibras. Os autores concluíram que os melhores resultados ocorreram quando o traço do concreto utilizado foi 1:3:3 (cimento:areia:brita) com fibra de comprimento aproximado de $30 \mathrm{~mm}$, espessura grossa (diâmetro de 0,30 a $0,35 \mathrm{~mm}$ ) e tratadas com água fervente; este resultado reforça a afirmação de que o comprimento crítico da argamassa obtido no presente estudo é próximo aos $25 \mathrm{~mm}$; já a determinação do comprimento crítico da fibra de coco é importante para garantir o melhor desempenho da argamassa e definir a aplicação específica visando à sua plena utilização na construção civil.

$\mathrm{Na}$ Figura 3 se constata a resistência à compressão superior da argamassa de referência nas três idades avaliadas (7, 28 e 56 dias). Esta queda de resistência das argamassas com adição de fibra de coco pode ser atribuída, de acordo com Savastano Júnior \& Agopyan (1999), ao fato das fibras vegetais apresentarem uma taxa de absorção de água superior a $80 \%$, o que inibe o efeito de parede e produz forte fluxo de água na direção da fibra provocando um aumento local da relação água/aglomerante elevando a porosidade na zona de transição entre a fibra e a matriz.

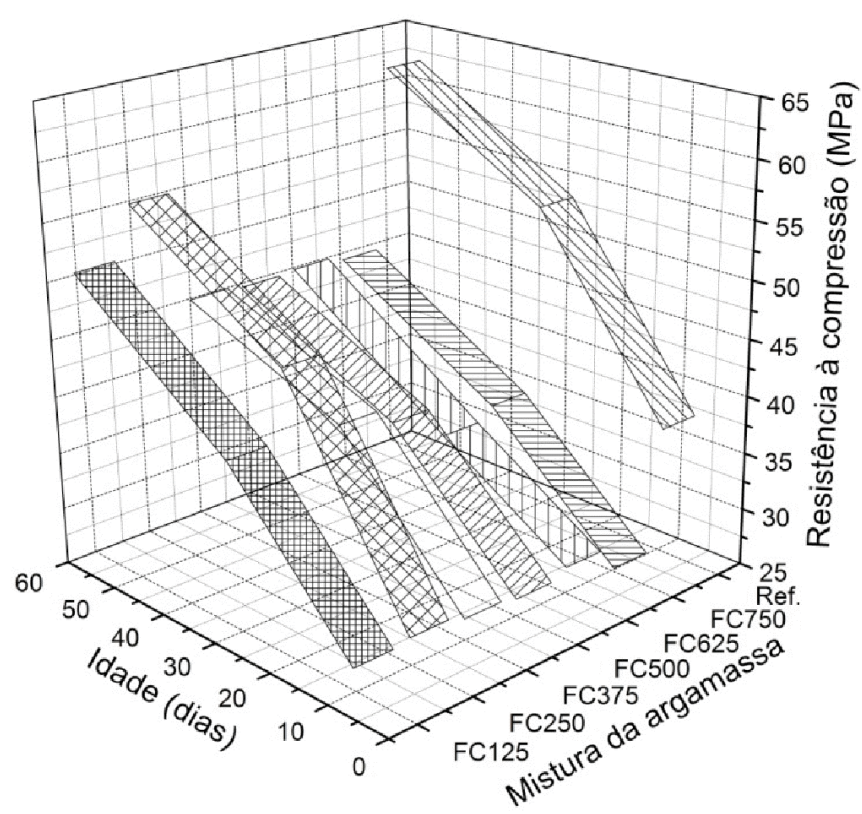

Figura 3. Resistência mecânica das argamassas com as três idades

Toledo Filho et al. (1997) encontraram uma redução da resistência à compressão da argamassa com fibra de coco em relação à de referência, de 18,4\% (para o teor adicionado de $2 \%$ ) e de $29 \%$ para o teor adicionado de fibra de $3 \%$. Os autores afirmam que, usando procedimentos convencionais de mistura, a adição de uma alta fração volumétrica de fibras à matriz introduz um número maior de defeitos em razão de uma compactação ineficiente da mistura fresca. Esses defeitos aumentam com o crescimento da quantidade de fibras uma vez que a mistura se torna mais rígida e menos trabalhável.

A maior porosidade da zona interfacial da argamassa fibrosa, também relacionada a uma compactação ineficiente da mistura fresca, contribui para a redução da resistência à compressão (Agopyan \& Savastano Júnior, 2003). As fibras de maior comprimento promovem efeito similar na mistura de argamassa, diminuindo seu desempenho.

Por outro lado, a rugosidade superficial das fibras de coco, particularmente as saliências dispostas como fileiras de pequenos nódulos, pode ajudar na adesão mecânica da matriz durante o processo de cura e assim melhora a tensão interfacial (Monteiro et al., 2006) mesmo que este efeito não tenha sido 
observado nas argamassas com comprimentos maiores de fibra de coco $(37,5 ; 50,0 ; 62,5$ e 75,0 mm).

Em relação ao comportamento observado após a ruptura do corpo de prova verifica-se, na Figura 4, um aumento da ductilidade do compósito pós-fissuração, quanto à argamassa de referência; na argamassa FC 25,0, por exemplo, as fibras mantiveram as faces das fissuras unidas não permitindo, assim, a perda da continuidade do material e evitando sua ruptura brusca, evidenciando a maior capacidade de deformação que a fibra promoveu na argamassa.

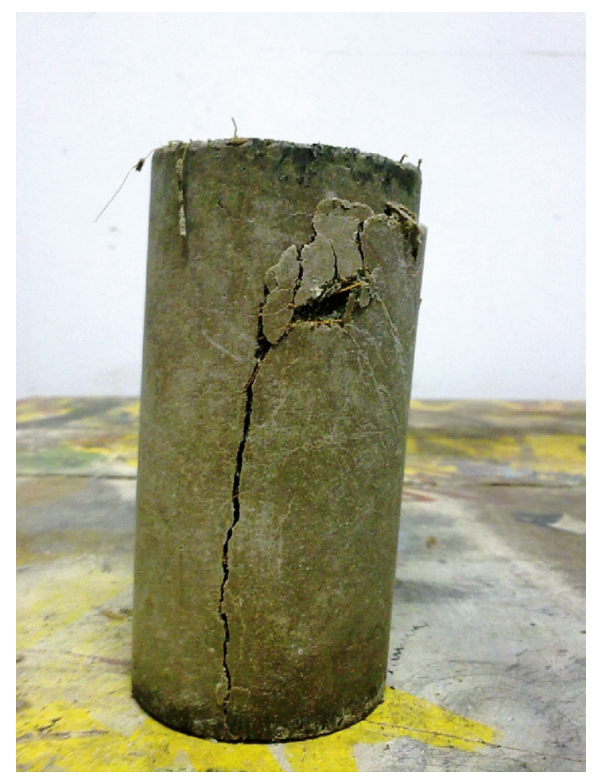

Figura 4. Corpo de prova de argamassa FC 25,0 rompido

Savastano Júnior \& Pimentel (2000) e Arsène et al. (2013) afirmam que após a fissuração da matriz, mesmo com redução significativa da força suportada, as fibras de coco mostraram capacidade suplementar de absorção de energia, em lugar do colapso instantâneo observado para os demais compósitos e matriz sem reforço.

Esta capacidade também é verificada por Toledo Filho et al. (1997) ao afirmarem que o corpo de prova (com fibra de coco) rompido indica que o modo de propagação da fissura foi por tração vertical; o aumento na resistência cisalhante, ocasionado pela presença das fibras, modificou o modo de ruptura da matriz; neste trabalho os corpos de prova reforçados com fibras não romperam em vários pedaços depois que a tensão de ruptura foi alcançada como a argamassa de referência, sem a presença da fibra de coco. Segundo Lima et al. (2014), as fibras de coco ainda atuam evitando o rompimento brusco do compósito devido à tensão de cisalhamento existente entre a matriz e a fibra.

É importante ressaltar que a diminuição da resistência à compressão não representa, necessariamente, um impeditivo para sua utilização. Considerando o valor mínimo de $20 \mathrm{MPa}$ para concretos estruturais da NBR-6118 (ABNT, 2003) observase que todas as argamassas com fibra de coco ultrapassam o limite mínimo de resistência; esses resultados apontam para a possibilidade técnica do uso de fibras de coco em argamassa na construção civil; além disto, não há uma legislação específica, por parte do CONFEA (Conselho Federal de Engenharia e Agronomia) que regulamente propostas neste sentido. Muitos autores (Silva et al., 2012; Ramli et al., 2013; Pereira et al., 2013; Lima et al., 2014) comparam o desempenho de matrizes com e sem a adição de resíduos para avaliar suas propriedades e, assim, verificar se o comportamento é adequado para a aplicação em engenharia.

\section{Conclusões}

1. O comprimento ótimo da fibra de coco para a argamassa estudada é próximo aos $25,0 \mathrm{~mm}$.

2. A adição de fibras de coco às matrizes de argamassa reduz a resistência a compressão, em comparação com a da argamassa de referência em aproximadamente 12\% (argamassa FC 25,0 na idade de 56 dias) a aproximadamente $40 \%$ (argamassa FC 75,0 na idade de 7 dias).

3. Os comprimentos maiores de fibra de coco diminuem a consistência da argamassa tornando a argamassa menos fluida.

4. A presença da fibra de coco na argamassa aumenta a capacidade de deformação dos corpos de prova permitindo uma ruptura menos brusca sob compressão.

\section{Literatura Citada}

Abdullah, A.; Jamaludin, S. B.; Anwar, M. I.; Noor, M. M.; Hussin, K. Assessment of Physical and Mechanical Properties of Cement Panel Influenced by Treated and Untreated Coconut Fiber Addition. Physics Procedia, v.22, p.263-269, 2011.

ABNT - Associação Brasileira de Normas Técnicas. NBR 7215: Cimento portland: Determinação da resistência à compressão. Rio de Janeiro: ABNT, 1996. 5p.

ABNT - Associação Brasileira de Normas Técnicas. NBR 6118: Projetos de estruturas de concreto: Procedimentos. Rio de Janeiro: ABNT, 2003. 225p.

Agopyan, V.; Savastano Júnior, H. Cinzas e aglomerantes alternativos. In: Freire, W. J; Beraldo, A. L. (Coordenadores) Tecnologias e materiais alternativos de construção. Campinas: UNICAMP, 2003. 331p.

Ali, M.; Li, Xiaoyang, Chouw, N. Experimental investigations on bond strength between coconut fibre and concrete, Materials and Design, v.44, p.596-605, 2013.

Ali, M.; Liu, A; Sou, H.; Chouw, N. Mechanical and dynamic properties of coconut fibre reinforced concrete. Construction and Building Materials, v.30, p.814-825, 2012.

Arsène, M. A.; Bilba, K.; Savastano Júnior, H.; Ghavami, K. Treatments of non-wood plant fibres used as reinforcement in composite materials. Materials Research, v.16, p.903-923, 2013.

Asasutjarit, C.; Hirunlabh, J.; Khedari, J.; Charoenvai, S.; Zeghmati, B.; Cheul Shin, U. Development of coconut coirbased lightweight cement board, Construction and Building Materials, v.21, p.277-288, 2007. 
Brígida, A. I. S.; Calado, V. M. A.; Gonçalves, L. R. B.; Coelho, M. A. Z.; Effect of chemical treatments on properties of green coconut fiber. Carbohydrate Polymers, v.79, p.832838, 2010.

Claramunt, J.; Ardanuy, M.; Hotal, J. A. G.; Toledo Filho, R. D. The hornification of vegetable fibers to improve the durability of cement mortar composites. Cement \& Concrete Composites, v.33, p.586-595, 2011.

Gunasekaran, K.; Kumar, P. S.; Lakshmipath, M. Mechanical and bond properties of coconut shell concrete. Construction and Building Materials, v.25, p.92-98, 2011.

Hassan, A.; Salema, A. A.; Ani, F. A.; Bakar, A. A. A review on oil palm empty fruit bunch fibre-reinforced polymer composite materials. Polymer Composites, v.31, p.20792101, 2010.

IBGE - Instituto Brasileiro de Geografia e Estatística. Levantamento sistemático da produção agrícola. Rio de Janeiro: IBGE, v.26, p.1-83, 2013.

Jhon, V. M.; Cincotto, M. A.; Sjostrom, C.; Agopyan, V.; Oliveira, C. T. A. Durability of slag mortar reinforced with coconut fibre. Cement \& Concrete Composites, v.27, p.565-574, 2005.

Li, Z.; Wang, L.; Wang. X. Flexural characteristics of coir fiber reinforced cementitious composites. Fibers and Polymers, v.7, p.286-294, 2006.

Lima, P. R. L.; Toledo Filho, R. D.; Melo Filho, J. A. Compressive stress-strain behaviour of cement mortar-composites reinforced with short sisal fibre. Materials Research, v.17, p.38-46, 2014.

Monteiro, S. N.; Terrones, L. A. H.; Carvalho, E. A.; Almeida, J. R. M. Efeito da interface fibra/matriz sobre a resistência de compósitos poliméricos reforçados com fibras de coco. Revista Matéria, v.11, p.395-402, 2006.

Pereira, C. L.; Savastano Júnior, H.; Payá, J.; Santos, S. F.; Borrachero, M. V.; Monzó, J.; Soriano, L. Use of highly reactive rice husk ash in the production of cement matrix reinforced with green coconut fiber. Industrial Crops and Products. v.49, p.88-96. 2013.

Ramires, A. R.; Castilho, F. C.; Domingues, V. J. M.; Gusman, M. $\mathrm{O}$. Thermal conductivity of coconut fibre filled ferrocement sandwich panels. Construction and Building Materials, v.37, p.425-431, 2012.
Ramli, M.; Kwan, W. H.; Abas, N. F. Strength and durability of coconut-fiber-reinforced concrete in aggressive environments. Construction and Building Materials, v.38, p.554-566, 2013.

Rodríguez, N. J., limón, M. Y.; miceli, F. A.; Gusman. O. G.; ortiz, T. P. M.; rivera, L. L.; Feijoo, J. A. V. Assessment of coconut fibre insulation characteristics and its use to modulate temperatures in concrete slabs with the aid of a finite element methodology. Energy and Buildings, v.43, p.1264-1272, 2011.

Savastano Júnior, H; Agopyan, V. Transition zone studies of vegetable fibre-cement paste composites. Cement \& Concrete Compósites, v. 21, p.49-57, 1999.

Savastano Júnior, H; Agopyan, V.; Nolasco, A. M. E.; Pimentel, L. Plant fibre reinforced cement components for roofing. Construction and Building Materials, v.13, p.433-438, 1999.

Savastano Júnior, H.; Pimentel, L. L. Viabilidade do aproveitamento de resíduos de Fibras vegetais para fins de obtenção de material de construção. Revista Brasileira de Engenharia Agrícola Ambiental. v.4, p.103-110, 2000.

Silva Filho, L. S. P.; Garcez, M. R. Compósitos de engenharia de matriz polimérica. In. Isaia, G. C. (ed.). Materiais de construção civil e princípios de ciência e engenharia de materiais. São Paulo: IBRACON, 2007. 1438p.

Silva, E. J.; Marques, M. L.; Fornari Júnior, C. Aplicação de fibra de coco em matrizes cimentícias. Revista Eletrônica em Gestão, Educação e Tecnologia Ambiental, v.8, p.1555$1561,2012$.

Toledo Filho, R. D. Materias compósitos reforçados com fibras naturais: Caracterização experimental. Rio de Janeiro: DEC-PUC/DEC, London: Imperial College. 1997. 472p. Tese Doutorado

Toledo Filho, R. D.; England, G. L.; Ghavami, K. Comportamento em compressão de argamassas reforçadas com fibras naturais. Revista Brasileira de Engenharia Agrícola Ambiental, v.1, p.79-88, 1997.

Toledo Filho, R. D.; Ghavami, K.; England, G. L.; Scrivener, K. Development of vegetable fibre-mortar composites of improved durability. Cement \& Concrete Composites, v.25, p.185-196, 2003.

Toledo Filho, R. D.; Scrivener, K.; England, G. L.; Ghavami, K. Durability of alkali-sensitive sisal and coconut fibres in cement mortar composites. Cement \& Concrete Composites, v.22, p.127-143, 2000. 